\title{
DE LOS CONTENIDOS A LAS COMPETENCIAS: APRENDER TRANSVERSALMENTE A TRAVÉS DEL CONOCIMIENTO SOCIAL*
}

\section{FROM CONTENTS TO COMPETENCES: TRANSVERSE LEARNING THROUGH SOCIAL KNOWLEDGE}

https://doi.org/10.15304/ie.30.6944

\author{
Francisco Rodríguez Lestegás \\ f.lestegas@usc.es \\ Xosé Carlos Macía Arce \\ carlos.macia@usc.es \\ Francisco X. Armas Quintá \\ francisco.armas@usc.es \\ Departamento de Didácticas Aplicadas \\ Universidade de Santiago de Compostela
}

\section{RESUMEN}

Este artículo aborda la contribución del conocimiento social escolar a la adquisición de aprendizajes transversales, bien sea a través de contenidos de gran relevancia social y fuerte componente actitudinal o a través de competencias clave. Para ello, se revisa tanto la legislación educativa española como la recomendación del Parlamento Europeo y del Consejo que insta a los Gobiernos de la Unión Europea a introducir la enseñanza y el aprendizaje de competencias clave en sus estrategias de aprendizaje permanente. En este contexto, se presta especial atención a las competencias sociales y cívicas, que implican la capacidad para situar el conocimiento social al servicio del funcionamiento democrático de la sociedad. No obstante, el enfoque metodológico centrado en las competencias clave que propugnan las disposiciones curriculares vigentes supone cambios de largo alcance en la concepción de la enseñanza y el aprendizaje, en la organización escolar y en la propia cultura profesional de los docentes. La búsqueda de aprendizajes transversales requiere nuevas formas de enseñar y aprender que superan los estrechos límites de las materias tradicionales, por lo que se concluye planteando la necesidad de desarrollar en el aula estrategias que posibiliten un trabajo docente colaborativo, un análisis reflexivo conjunto de los criterios de evaluación y de los estándares de aprendizaje, y un continuo intercambio de información sobre el proceso de aprendizaje del alumnado.

Palabras clave: Temas transversales; Competencias clave; Competencias sociales y cívicas; Ciencias Sociales

Recibido: 17NI/2020. Aceptado: IX/2020

* Artículo realizado en el marco del proyecto de investigación PGC2018-094491-B-C31. Convocatoria 2018 del Plan Nacional de I+D+i y Fondos FEDER 


\begin{abstract}
This paper deals with the contribution of school social knowledge to the acquisition of transversal learning, either through contents of great social relevance and strong attitude component or through key competences. To do so, both the Spanish educational legislation and the recommendation of the European Parliament and of the Council that urges the governments of the European Union to introduce the teaching and learning of key competences in their lifelong learning strategies are reviewed. In this context, special attention is given to social and civic competences, which imply the ability to put social knowledge at the disposal of the democratic functioning of society. However, the methodological perspective based on key competences, which today shape a growing number of curricula, implies particular teaching approaches as well as changes to school organisation and professional culture. The search for transversal learning requires new ways of teaching and learning that exceed the narrow limits of traditional subjects. Therefore, the paper concludes by stating the need to develop strategies in the classroom that enable a collaborative teaching work, a joint discussion regarding assessment standards, and a continuous exchange of information about the learning development of students.
\end{abstract}

Keywords: Cross-curricular subjects; Key competences; Social and civic competences; Social Sciences

\title{
INTRODUCCIÓN
}

El proceso de institucionalización y burocratización de la enseñanza, iniciado a finales del siglo XVIII con el reconocimiento del derecho a la educación, supuso el desarrollo de sistemas educativos nacionales y, con ello, la configuración de las materias escolares como el núcleo alrededor del cual comenzó a girar la escolarización de un alumnado en continua expansión. En este contexto, las asignaturas han adquirido la naturaleza de construcciones particulares, muchas veces al margen de las ciencias de referencia, encargadas de vehicular lo que Forquin (1988) denomina "cultura escolar", constituida por un detallado conjunto de conocimientos, competencias, actitudes y valores que la escuela se encarga de transmitir explícita o implícitamente a los estudiantes como bagaje cultural y patrimonio común para todos los ciudadanos.

De este modo, cada materia tiene como misión aportar un contenido instructivo que se pone al servicio del objetivo que la sociedad asigna a las instituciones escolares, y que no es otro que transmitir a los estudiantes una determinada representación del mundo en el que viven, lo que convierte a la escuela en un cauce preferente de transferencia intergeneracional (Audigier, 1997). Esto hace que, en principio, las asignaturas se legitimen por sí mismas, dado que sirven para aprender los contenidos y resolver los ejercicios etiquetados como característicos de tales materias de enseñanza. Son un compendio de conocimientos que, sin discusión, es preciso adquirir porque la sociedad así lo ha decidido, en virtud de la utilidad que se les atribuya. Y para ello, las materias escolares, más que una simple suma de conocimientos pretendidamente científicos, consisten en compartimentos de saberes que determinan todos los aspectos de la organización escolar y la relación del alumnado con el propio conocimiento que aprenden (Chervel, 1991, 1998; Rodríguez Lestegás, 2000, 2002, 2003). 


\section{EN BUSCA DE LA TRANSVERSALIDAD: LA CONTRIBUCIÓN DE LAS CIENCIAS SOCIALES A LAS "DIMENSIONES TRANSVERSALES AL CURRÍCULO"}

A la hora de estructurar los contenidos, la $\operatorname{LOGSE}^{1}$ adoptó por vez primera el concepto de "área" como unidad e instrumento articulador de los currículos propios del amplio período correspondiente a la enseñanza que esa disposición legal consideraba básica, obligatoria y gratuita (educación primaria y educación secundaria obligatoria), así como de la educación infantil. Con este criterio se buscaba un marco organizativo lo suficientemente amplio y flexible como para superar las limitaciones impuestas por las disciplinas escolares tradicionales estrechamente vinculadas a la especialización académica y científica, al tiempo que adaptado a la configuración de una educación desarrollada bajo los principios de comprensividad y atención a la diversidad. Sin embargo y pese a tan loables propósitos, el inevitable peso de la fuente disciplinar va ganando progresivamente importancia a lo largo de la educación primaria y secundaria obligatoria, para imponerse definitivamente en el bachillerato.

Ante esta situación, el desarrollo normativo de la LOGSE planteó la primera apuesta a favor de la "transversalidad". Así, en los respectivos preámbulos (luego reiterado en el articulado) de los reales decretos por los que se establecen los currículos de la educación primaria y de la educación secundaria obligatoria ${ }^{2}$ puede leerse:

El carácter integral del currículo significa también que a él se incorporan elementos educativos básicos que han de integrarse en las diferentes áreas y que la sociedad demanda, tales como la educación para la paz, para la salud, para la igualdad entre los sexos, educación ambiental, educación sexual, educación del consumidor y educación vial. (Real Decreto 1344/1991 y Real Decreto 1345/1991).

Se trata de una aportación supuestamente novedosa con la finalidad de que en las distintas áreas y materias estén presentes determinadas dimensiones transversales al currículo, que "hacen referencia a los problemas y conflictos, de gran trascendencia, que se producen en la época actual y frente a los que resulta urgente una toma de posiciones personal y colectiva" (Reyzábal y Sanz, 1995, p. 15). Montserrat Moreno ya había enunciado estos contenidos, no contemplados en las disciplinas clásicas, que era necesario tratar transversalmente

porque vivimos en una sociedad que está clamando por la paz, por la igualdad de derechos y oportunidades entre el hombre y la mujer, por una conservación y mejora del medio ambiente, por vivir de una manera más saludable, por un desarrollo de la afectividad y la sexualidad que permita mejorar las relaciones interpersonales; una sociedad que necesita forjar personalidades autónomas y críticas, capaces de respetar la opinión de los demás y, a la vez, de defender sus derechos. (Moreno, 1993, p. 22).

Ley Orgánica 1/1990, de 3 de octubre, de Ordenación General del Sistema Educativo (BOE, 4 octubre 1990). https://www.boe.es/boe/dias/1990/10/04/pdfs/A28927-28942.pdf

${ }^{2}$ Real Decreto 1344/1991, de 6 de septiembre, por el que se establece el currículo de la Educación Primaria (BOE, 13 septiembre 1991) y Real Decreto 1345/1991, de 6 de septiembre, por el que se establece el currículo de la Educación Secundaria Obligatoria (BOE, 13 septiembre 1991).

https://www.boe.es/boe/dias/1991/09/13/pdfs/A30226-30228.pdf

https://www.boe.es/boe/dias/1991/09/13/pdfs/A30228-30231.pdf 
Se trata, como vemos, de contenidos que habían adquirido un notable desarrollo en aquellos momentos, debido precisamente a su gran relevancia social, y que presentan un fuerte componente actitudinal, pero que no pertenecen propiamente al ámbito de ninguna disciplina concreta, sino que, con una presencia diferente en cada caso, precisan de la aportación conceptual de las distintas áreas o materias curriculares para su correcto tratamiento didáctico. De este modo, las dimensiones transversales no se sitúan en paralelo a las áreas curriculares, sino que las "atraviesan" o "impregnan" recíprocamente, lo que significa que los contenidos transversales están presentes en las áreas y éstas se hallan también presentes en aquéllos, a la vez que las diferentes temáticas transversales se interrelacionan entre sí (Rodríguez Lestegás, 1994, 1997).

La presencia de los ejes transversales en el currículo no suponía, pues, la incorporación de nuevas asignaturas al mismo, sino una "llamada de atención" (González Lucini, 1994), una nueva perspectiva, un enfoque diferente de la realidad a partir de los contenidos propios de las diversas áreas y disciplinas académicas. No obstante, los temas transversales, configurados inicialmente como grandes ejes de globalización e interdisciplinariedad, fueron girando progresivamente hacia una educación en valores en relación con los contenidos actitudinales que proponía el currículo.

\subsection{Ciencias Sociales y educación para la paz}

La educación para la paz es una dimensión continua y permanente del sistema educativo, por lo cual afecta, no solo a todas las disciplinas, sino incluso a todos los elementos que componen la estructura escolar. Cada vez en mayor medida, es necesario aprovechar cualquier ocasión para fomentar la toma de conciencia ante las relaciones sociales que promueven la violencia.

De hecho, hemos sido acostumbrados a minimizar la importancia de la paz como elemento histórico, dado que siempre son los dominantes y victoriosos quienes protagonizan la vida y el devenir de los pueblos. Los héroes que destacan los textos escolares suelen ser audaces militares, poderosos políticos, valientes revolucionarios. Se ha promocionado la idea de que la guerra ha desempeñado un papel dinámico en el transcurso de la historia, por lo que es muy difícil encontrar un héroe que se haya distinguido por su actividad en favor de la paz (Lederach, 1984).

En este contexto, las Ciencias Sociales deben alertar ante las situaciones de etnocentrismo, desigualdad, marginación, discriminación, xenofobia, racismo, segregación, hostilidad y violencia. Frente a ello, la educación para la comprensión internacional e intercultural, para la defensa de los derechos humanos, para la tolerancia de la diversidad ideológica, para el compromiso con la justicia social y la solidaridad humana, para la cooperación y el desarrollo, para el desarme, para la solución de conflictos, son algunos de los componentes que conforman la educación para la paz y que pueden ser abordados desde el conocimiento social (Rodríguez Jares, 1991). 


\subsection{Ciencias Sociales y educación afectivo-sexual}

Parece evidente que la realidad social no puede entenderse en su totalidad sin tener en consideración la sexualidad, pero ésta tampoco puede comprenderse al margen de los fenómenos y procesos sociales. A este respecto, Barragán nos dice que

la sexualidad humana explica procesos sociales tan importantes como la construcción de la identidad sexual; la identidad de género; los roles; la vinculación afectiva; relaciones como la amistad y el amor; instituciones como la familia, el matrimonio o el divorcio y funciones como la comunicación, el placer o la reproducción. (Barragán, 1991, p. 52).

Numerosas investigaciones antropológicas y etológicas han puesto de relieve que el ser humano puede manifestar multitud de conductas sexuales diferentes, de las cuales la cultura se encarga de seleccionar unas y condenar otras. No obstante, los currículos suelen estar diseñados en torno a una concepción biologicista, genitalista, reproductiva e individualista de la sexualidad humana, subestimando sus componentes socioculturales.

Sin embargo, nada más lejos de la realidad, puesto que todos los modelos de sexualidad humana han sido construidos históricamente, de manera que los fenómenos que se derivan de ella no existirían al margen de la voluntad de los seres humanos. Así, pues, el conocimiento afectivo-sexual es, por lo menos, tan social como biológico, dado que incluso los aspectos claramente fisiológicos, como ocurre con la función reproductora, están regulados socialmente y así es como son percibidos por los alumnos y alumnas (Barragán, 1991).

\subsection{Ciencias Sociales e igualdad de oportunidades entre las personas de am- bos sexos}

Es sabido que los estereotipos de sexo/género ${ }^{3}$, tan arraigados en las creencias populares, han llevado a la aceptación general de que hombres y mujeres son básicamente diferentes y que, desde luego, la mujer es inferior al hombre. Este "androcentrismo" (Moreno, 1986), que impregna todos los aspectos de la vida y en el que participan tanto hombres como mujeres, ha calado hondo en la enseñanza, tarea en la que ha desempeñado un papel decisivo el contenido marcadamente sexista de los libros de texto.

Las Ciencias Sociales, fuertemente ideologizadas y con unos contenidos demasiadas veces sesgados y tendenciosos, se han venido mostrando como un arma altamente eficaz para inculcar en la infancia y en la juventud una concepción androcéntrica del mundo. Así, escolares de ambos sexos imitan los modelos fuertemente sexistas transmitidos, tanto a través del currículo explícito como del oculto, por la escuela, por el profesorado, así como por los libros y otros materiales utilizados en las clases.

3 El "sexo" es una categoría biológica que, a partir de las diferencias fisiológicas y morfológicas, permite dividir a los seres humanos entre "hombres" y "mujeres". El "género", por su parte, es una construcción sociocultural en virtud de la cual se asignan a mujeres y hombres características, atributos, expectativas, comportamientos y roles que permiten establecer patrones "femeninos" y "masculinos". 
La contribución del conocimiento social a la transformación positiva de la mentalidad sexista se sitúa fundamentalmente en el ámbito procedimental y actitudinal: identificación de situaciones de discriminación y marginación por razón de sexo; uso no sexista del lenguaje; análisis crítico de la utilización publicitaria de los estereotipos masculino y femenino; establecimiento de relaciones cooperativas y respetuosas entre personas de ambos sexos (Reyzábal y Sanz, 1995).

\subsection{Ciencias Sociales y educación ambiental}

La Naturaleza ha sido considerada durante mucho tiempo como un conjunto de bienes al servicio del desarrollo humano y, por lo tanto, un recurso para ser explotado como si fuese inagotable. Pero ahora, cuando se conoce mejor que nunca y comienza a padecerse la fragilidad y vulnerabilidad de nuestro planeta, ya no es posible seguir degradando irreversiblemente el territorio haciendo caso omiso de las proclamas a favor de un desarrollo sostenible.

En el plano educativo, la concepción de una humanidad depredadora ha dado paso a una nueva sensibilidad ante el impacto ambiental producido por nuestras acciones sobre el espacio, lo que ha ido abriendo camino a una educación ambiental que tiene por objetivo "conseguir que las personas desarrollen conductas correctas respecto a su entorno de acuerdo con valores asumidos libre y responsablemente" (Novo, 1995, p. 107).

Nuestro país no ha permanecido ajeno al importante desarrollo que en el panorama internacional ha adquirido la educación ambiental, convertida en una de las dimensiones formativas mejor valoradas por la ciudadanía. Ahora bien, este interés tan notable que la educación ambiental suscita en la actualidad está estrechamente vinculado a la efervescencia de la alarma ecologista y a la emergencia climática, lo que prioriza la dimensión ecológica y ambientalista del problema en detrimento de otras consideraciones estrechamente relacionadas con las desigualdades sociales y los desequilibrios territoriales. Por eso se puede afirmar que la degradación del medio constituye un magnífico instrumento para conocer la realidad social (Sanz López y Sánchez Alhama, 1995).

Desde esta consideración, es preciso defender que las Ciencias Sociales pueden aportar propuestas metodológicas integradoras particularmente adecuadas para el estudio de los problemas ambientales, en las que cabe destacar el papel de referencia central asignado a hombres y mujeres, lo que Muñoz Jiménez (1992, p. 5) denomina "perspectiva ecológica antropocéntrica”. En efecto, no debe perderse de vista que los graves problemas ecológicos, tal como hoy los percibimos y sufrimos a escala local y global, tienen una causalidad específicamente humana. Al mismo tiempo, nuestra especie es la única que tiene historia, que crea civilización, que es capaz de progresar socialmente, que dispone de cultura y que es capaz de transmitirla de generación en generación a través de la educación.

Pues bien, si el género humano es el único capaz de alterar el equilibrio ecológico del planeta mediante sus agresiones al medio físico y también el único que puede generar cultura y educación, la consecuencia más apreciable de esta doble afirmación es que el problema medioambiental, además de científico, es de índole social y cultural, manifestándose y percibiéndose de distinta forma según las épocas históricas, según los niveles de civilización alcanza- 
dos por los pueblos y según la influencia de factores diversos, como pueden ser los geográficos, históricos, políticos, sociales, económicos y educativos (Clemente, 1992).

\subsection{Ciencias Sociales y educación del consumidor}

La "sociedad de consumo", en la que se desenvuelve nuestra existencia, ha sido caracterizada como "una sociedad marcada por un fuerte desequilibrio entre un frente constituido por productores y otro integrado por los consumidores" (Pujol, 1996, p. 18); y en esta situación, la necesidad de proteger o defender a los consumidores y usuarios, muchas veces en clara situación de indefensión en un mercado dominado por las empresas suministradoras de productos y servicios, es cada vez más manifiesta. Pues bien, dentro de la masa potencialmente consumidora, la población en edad escolar constituye un grupo especialmente atractivo: primero, porque los niños y jóvenes adquieren o son inducidos a la compra de numerosos productos; segundo, porque, a la vez, inducen al consumo a aquellos adultos con los que conviven; y tercero, porque representan una magnífica opción de futuro, una vez que se logre despertar en ellos un considerable número de necesidades y fomentar los correspondientes hábitos de consumo (Cainzos, 1993).

Es cierto que la extensión de las posibilidades consumistas en los países desarrollados ha permitido un notable incremento de la calidad de vida de muchos de sus habitantes, pero no lo es menos que, al mismo tiempo, ha sumido en la pobreza a muchos otros países, aumentando las desigualdades y el contraste entre ricos y pobres hasta límites cada vez más insoportables (Pujol, 1996). De ahí la necesidad de una adecuada educación del consumidor que no se reduzca a enseñar a los estudiantes a rentabilizar al máximo su dinero en el momento de efectuar una compra (posición "liberal"), ni tampoco a proporcionarles la formación necesaria para que sean capaces de analizar críticamente las actitudes consumistas (posición "reformista"), sino que debe incluir además la posibilidad de dotar a los alumnos y a las alumnas de una serie de habilidades cognitivas, sociales, emocionales, cívicas y conductuales que les sirvan para responsabilizarse en relación con las posibles consecuencias que sus actuaciones consumistas tienen en la sociedad y sobre el medio ambiente (posición "responsable"), sin olvidar la consideración de los hábitos consumistas en el contexto de una sociedad radicalmente injusta que permite la explotación de la mayoría a manos de una minoría (posición "radical) (Cidad, 1991; Pujol, 1996).

\subsection{Ciencias Sociales y educación para la salud}

Aunque la salud es considerada en todas las culturas un bien indispensable que es necesario preservar o recuperar, en la consideración de este concepto intervienen conocimientos, tradiciones, creencias, valores y normas que convierten la idea de salud en una construcción social de carácter dinámico, puesto que es propia de cada cultura y momento histórico.

Entre nosotros, todavía es habitual identificar la salud con la ausencia de enfermedad, lo que hace que la sociedad demande soluciones rápidas y eficaces para dolencias que pueden tener graves consecuencias, como son el cáncer, las enfermedades cardiovasculares, los trastornos metabólicos y degenerativos, y las infecciones cuya transmisión es difícil de controlar 
(Reyzábal y Sanz, 1995). Sin embargo, ya desde 1946 la Organización Mundial de la Salud la define como "un estado de completo bienestar físico, mental y social, y no solamente la ausencia de afecciones o enfermedades" ". Se trata, pues, de un concepto complejo que, junto a la dimensión puramente individual (biológica y mental), incorpora también determinados factores socioambientales, tales como la higiene, la alimentación, la educación, el nivel de ingresos y posición social, la vivienda, el empleo y las condiciones laborales, los entornos sociales, el estrés, el ruido o el deterioro ecológico (Busquets y Leal, 1993).

En las últimas décadas, la presión social se ha centrado en la necesidad de evitar situaciones de riesgo para la población, como es el caso de las drogodependencias, las enfermedades de transmisión sexual o la prevención de embarazos y accidentes. En estos momentos, la crisis provocada por el COVID-19 ha puesto de relieve la utilidad de las ciencias sociales para interpretar la complejidad de los fenómenos y procesos que ocurren a nuestro alrededor: las consecuencias de la globalización y los flujos internacionales, el impacto de la degradación medioambiental, la trascendencia de las relaciones geopolíticas, las repercusiones de la actividad económica, los efectos de la desigual distribución de la población y los comportamientos cotidianos de las personas.

En concreto, el conocimiento geográfico e histórico nos señala lo que podemos aprender de las otras grandes pandemias del pasado (la peste negra de mediados del siglo XIV, el cólera del siglo XIX o la gripe "española” de 1918-1919), en lo que se refiere a su origen, su expansión, el pánico desatado entre las gentes, las medidas adoptadas desde el poder para frenar el contagio y las repercusiones sanitarias, demográficas y económicas.

\section{UN CAMBIO DE PERSPECTIVA: APRENDER TRANSVERSALMENTE A TRAVÉS DE LAS COMPETENCIAS SOCIALES Y CÍVICAS}

$\mathrm{La} \mathrm{LOE}^{5}$, aun manteniendo el tratamiento transversal de la educación en valores y otras enseñanzas, incorpora la novedad de incluir las competencias básicas entre los componentes del currículo, junto a los objetivos, contenidos, métodos pedagógicos y criterios de evaluación. La definición de estas competencias básicas adquiere especial relevancia en la regulación de las enseñanzas mínimas ${ }^{6}$ que el alumnado deberá desarrollar a lo largo de la educación primaria

4 Preámbulo de la Constitución de la OMS, aprobada en la Conferencia Internacional de la Salud celebrada en Nueva York en 1946 y que entró en vigor en 1948. https://www.who.int/es/about/who-weare/constitution

5 Ley Orgánica 2/2006, de 3 de mayo, de Educación (BOE, 4 mayo 2006). https://www.boe.es/boe/dias/2006/05/04/pdfs/A17158-17207.pdf

6 Real Decreto 1513/2006, de 7 de diciembre, por el que se establecen las enseñanzas mínimas de la Educación primaria (BOE, 8 diciembre 2006) y Real Decreto 1631/2006, de 29 de diciembre, por el que se establecen las enseñanzas mínimas correspondientes a la Educación Secundaria Obligatoria (BOE, 5 enero 2007).

https://www.boe.es/boe/dias/2006/12/08/pdfs/A43053-43102.pdf

https://www.boe.es/boe/dias/2007/01/05/pdfs/A00677-00773.pdf 
y alcanzar una vez finalizada la educación secundaria obligatoria, permitiendo "identificar aquellos aprendizajes que se consideran imprescindibles desde un planteamiento integrador y orientado a la aplicación de los saberes adquiridos".

En el Anexo I de las diferentes disposiciones que regulan las enseñanzas mínimas de cada etapa se insiste en el papel central que cumplen las competencias básicas en relación con la integración de los aprendizajes:

La inclusión de las competencias básicas en el currículo tiene varias finalidades. En primer lugar, integrar los diferentes aprendizajes, tanto los formales, incorporados a las diferentes áreas o materias, como los informales y no formales. En segundo lugar, permitir a todos los estudiantes integrar sus aprendizajes, ponerlos en relación con distintos tipos de contenidos y utilizarlos de manera efectiva cuando les resulten necesarios en diferentes situaciones y contextos. Y, por último, orientar la enseñanza, al permitir identificar los contenidos y los criterios de evaluación que tienen carácter imprescindible y, en general, inspirar las distintas decisiones relativas al proceso de enseñanza y de aprendizaje. (Real Decreto 1513/2006 y Real Decreto 1631/2006).

En síntesis, las competencias básicas que introduce la LOE tienen un carácter interdisciplinar y transversal, ya que integran aprendizajes procedentes de diversas áreas y materias académicas, al tiempo que combinan contenidos de los ámbitos cognoscitivo-declarativo (saber decir), procedimental (saber hacer) y actitudinal (saber ser), y promueven su utilización en situaciones determinadas y contextos específicos (aplicabilidad y transferencia).

Posteriormente, la $\mathrm{LOMCE}^{7}$ trata de ir más allá al poner el énfasis en un modelo de currículo basado en competencias, que define como "capacidades para aplicar de forma integrada los contenidos propios de cada enseñanza y etapa educativa, con el fin de lograr la realización adecuada de actividades y la resolución eficaz de problemas complejos". La legislación estatal de desarrollo de la LOMCE que establece el currículo básico ${ }^{8}$ ofrece la oportunidad de ampliar esta definición:

La competencia supone una combinación de habilidades prácticas, conocimientos, motivación, valores éticos, actitudes, emociones, y otros componentes sociales y de comportamiento que se movilizan conjuntamente para lograr una acción eficaz. Se contemplan, pues, como conocimiento en la práctica, un conocimiento adquirido a través de la participación activa en prácticas sociales que, como tales, se pueden desarrollar tanto en el contexto educativo formal, a través del currículo, como en los contextos educativos no formales e informales. (Real Decreto 126/2014 y Real Decreto 1105/2014).

7 Ley Orgánica 8/2013, de 9 de diciembre, para la mejora de la calidad educativa (BOE, 10 diciembre 2013). https://www.boe.es/boe/dias/2013/12/10/pdfs/BOE-A-2013-12886.pdf

8 Real Decreto 126/2014, de 28 de febrero, por el que se establece el currículo básico de la Educación Primaria (BOE, 1 marzo 2014) y Real Decreto 1105/2014, de 26 de diciembre, por el que se establece el currículo básico de la Educación Secundaria Obligatoria y del Bachillerato (BOE, 3 enero 2015). https://www.boe.es/boe/dias/2014/03/01/pdfs/BOE-A-2014-2222.pdf https://www.boe.es/boe/dias/2015/01/03/pdfs/BOE-A-2015-37.pdf 
Se insiste, además, en que el proceso de enseñanza-aprendizaje competencial "se caracteriza por su transversalidad, su dinamismo y su carácter integral", por lo que "debe abordarse desde todas las áreas de conocimiento, y por parte de las diversas instancias que conforman la comunidad educativa” (Real Decreto 126/2014 y Real Decreto 1105/2014).

La LOMCE se apoya expresamente en una recomendación del Parlamento Europeo y del Consejo ${ }^{9}$, que insta a los Gobiernos de la Unión Europea a introducir la enseñanza y el aprendizaje de competencias clave en sus estrategias de aprendizaje permanente. En el marco de referencia europeo ${ }^{10}$ se definen las competencias como "una combinación de conocimientos, capacidades y actitudes adecuadas al contexto. Las competencias clave son aquéllas que todas las personas precisan para su realización y desarrollo personales, así como para la ciudadanía activa, la inclusión social y el empleo" (Comisión Europea, 2007, p. 3).

El documento establece ocho competencias clave esenciales para el bienestar de las sociedades europeas, el crecimiento económico y la innovación, que la LOMCE sintetiza en siete $^{11}$. La ley declara conceder especial atención a la adquisición de las "Competencias sociales y cívicas", al incluirlas "en la dinámica cotidiana de los procesos de enseñanza y aprendizaje" con el fin de potenciar "a través de un planteamiento conjunto, su posibilidad de transferencia y su carácter orientador". No obstante, esta loable intención no se materializa en los currículos básicos, en donde se indica reiteradamente que las competencias cuyo desarrollo se potenciará son "Comunicación lingüística" y "Competencia matemática y competencias básicas en ciencia y tecnología".

\subsection{Las competencias sociales y cívicas}

Las competencias sociales y cívicas implican la capacidad para situar el conocimiento social al servicio del funcionamiento democrático de la sociedad. Más en concreto, las competencias sociales (personales, interpersonales e interculturales) recogen conocimientos, capacidades y actitudes que habilitan a la personas "para participar de una manera eficaz y constructiva en la vida social y profesional, especialmente en sociedades cada vez más diversificadas" (Comisión Europea, 2007, p. 9). Por su parte, las competencias cívicas preparan a las personas "para participar plenamente en la vida cívica gracias al conocimiento de conceptos y estructuras sociales y políticas, y al compromiso de participación activa y democrática" (Comisión Europea, 2007, p. 9). En síntesis, las competencias sociales y cívicas permiten comprender la

9 Recomendación del Parlamento Europeo y del Consejo de 18 de diciembre de 2006 sobre las competencias clave para el aprendizaje permanente (2006/962/CE).

https://eur-lex.europa.eu/legal-content/ES/TXT/PDF/?uri=CELEX:32006H0962\&from=EN

10 Anexo a la Recomendación 2006/962/CE sobre las competencias clave para el aprendizaje permanente, publicado en Comisión Europea (2007).

11 Unifica las competencias "Comunicación en la lengua materna" y "Comunicación en lenguas extranjeras" en "Comunicación lingüística". Las restantes competencias clave son: "Competencia matemática y competencias básicas en ciencia y tecnología", "Competencia digital", "Aprender a aprender", "Competencias sociales y cívicas", "Sentido de iniciativa y espíritu emprendedor" y "Conciencia y expresiones culturales". 
sociedad en que se vive, afrontar los problemas y conflictos de convivencia que pueden surgir en ella, y ejercer la ciudadanía democrática de una forma activa.

Algunas competencias sociales se relacionan con el bienestar personal y colectivo, lo que exige entender cómo las personas pueden procurarse un estado de salud física y mental óptimo, y ser conscientes, por lo tanto, de la conveniencia de mantener estilos de vida saludables. La participación en los ámbitos interpersonal, social y profesional requiere comprender los códigos de conducta y los usos comúnmente aceptados en los diferentes entornos y sociedades. Estas competencias también se refieren al reconocimiento de la igualdad y no discriminación entre hombres y mujeres, culturas y grupos sociales; a la comprensión de las dimensiones multicultural y socioeconómica de las sociedades europeas, y a la gestión de las interacciones entre identidades nacionales e identidad europea.

Las competencias sociales incluyen las capacidades y actitudes de comunicación en distintos contextos y entre diferentes culturas, expresión de las propias opiniones y comprensión de puntos de vista ajenos al propio, tolerancia, negociación, colaboración, integridad, honestidad, respeto y empatía.

Por su parte, las competencias cívicas se refieren al conocimiento de los conceptos de democracia, libertad, justicia, igualdad, solidaridad, ciudadanía y derechos reconocidos en la legislación nacional y en las declaraciones internacionales, atendiendo a su aplicación por parte de las instituciones a distintas escalas. Junto al conocimiento de los principales acontecimientos y procesos históricos, así como los movimientos sociales y políticos, se requiere, en particular, disponer de información sobre el desarrollo de la integración europea y las estructuras resultantes, siendo conscientes, al mismo tiempo, de la diversidad e identidades culturales de Europa.

Las competencias cívicas están relacionadas con las capacidades de interactuar eficazmente en el ámbito público y de resolver los problemas que afecten a la comunidad. Esto supone la formación de ciudadanos reflexivos, críticos y creativos, capaces de participar constructivamente en las actividades de la comunidad y en la adopción de decisiones democráticas que competen al ejercicio de una ciudadanía participativa. Y todo ello desde la consideración de los valores de igualdad y diversidad, el respeto a la diferencia y la lucha contra la desigualdad, lo que implica el desarrollo de una construcción adecuada del sentimiento de identidad y pertenencia que sea compatible con el respeto a los valores compartidos imprescindibles para garantizar la cohesión de la comunidad (Comisión Europea, 2007).

\subsection{Nueva perspectiva, ¿nuevas prácticas?}

En origen, coincidiendo con el impacto de la globalización y la sociedad de la información y con el apogeo de las políticas neoliberales impulsadas desde gobiernos nacionales y organismos internacionales, la "educación por competencias" ha suscitado un notable rechazo en la comunidad educativa y, si nos referimos concretamente a las competencias sociales y cívicas, no ha facilitado el desarrollo de comportamientos científico-didácticos vinculados con la justicia social y espacial (Macía-Arce, Rodríguez-Lestegás y Armas-Quintá, 2018). Actualmente, 
el sesgado marco conceptual inicial parece haberse ensanchado y matizado para proponer una pedagógicamente más justificable "educación orientada al desarrollo de competencias".

Ciertamente, el enfoque metodológico centrado en las competencias clave que propugnan las disposiciones curriculares vigentes supone cambios de largo alcance en la concepción de la enseñanza y el aprendizaje, en la organización escolar y en la propia cultura profesional de los docentes. Las competencias transversales exigen nuevas formas de enseñar y aprender que superan los estrechos límites de las materias tradicionales. La búsqueda de aprendizajes transversales requiere un trabajo docente colaborativo, un análisis reflexivo conjunto de los criterios de evaluación y de los estándares de aprendizaje, y un continuo intercambio de información sobre el proceso de aprendizaje del alumnado (Comisión Europea/EACEA/Eurydice, 2012).

En nuestro país, se han dictado instrucciones que ayuden a entender las relaciones entre las competencias, los contenidos y los criterios de evaluación, al tiempo que se han propuesto orientaciones para facilitar el diseño y desarrollo de estrategias metodológicas que permitan trabajar por competencias en el aula ${ }^{12}$. Se trata de promover y evaluar las competencias desde las etapas educativas iniciales hasta su consolidación al final de los estudios preuniversitarios. Cabe entender que todas las áreas y materias deben contribuir al desarrollo competencial y que su evaluación requiere la utilización de instrumentos que permitan valorar los logros del alumnado en función de sus desempeños en la resolución de problemas que simulen situaciones reales.

Sin embargo, una cosa debe quedar clara: no es posible aprender transversalmente desde una conceptualización de la práctica escolar como compartimentos estancos en los que cada materia es un coto privado y cada docente permanece celosamente atrincherado en su dominio profesional. De ahí que las dificultades a este respecto se hacen difícilmente superables en el caso de la educación secundaria, en donde el currículo se organiza por materias y los docentes son (y se consideran) especialistas en un único ámbito académico. Revertir esta situación requeriría un considerable esfuerzo en la formación inicial y permanente del profesorado, así como la disponibilidad de recursos humanos y materiales necesarios para facilitar la reflexión, el debate y el trabajo conjunto en los centros escolares, pero también un cambio de actitud por parte de los profesionales de la educación que por el momento es difícil de avistar.

Cuando el currículo se organiza disciplinarmente y, en cambio, el desarrollo de las competencias transversales es una responsabilidad compartida de todos los docentes, se corre el serio riesgo de que nadie se ocupe de ello en la realidad del aula o de que cada quien lo interprete a su manera y lo evalúe desde su propia perspectiva. Por el contrario, la adquisición eficaz de las competencias básicas requiere que el profesorado diseñe "actividades de aprendizaje integradas que permitan a los alumnos avanzar hacia los resultados de aprendizaje de más de una competencia al mismo tiempo" (Comisión Europea/EACEA/Eurydice, 2012, p. 28). Por ejem-

12 Orden ECD/65/2015, de 21 de enero, por la que se describen las relaciones entre las competencias, los contenidos y los criterios de evaluación de la educación primaria, la educación secundaria obligatoria y el bachillerato (BOE, 29 enero 2015).

https://www.boe.es/boe/dias/2015/01/29/pdfs/BOE-A-2015-738.pdf 
plo, suele escucharse que muchos alumnos son incapaces de resolver los problemas de matemáticas simplemente porque no entienden los enunciados que se les plantean; en este escenario, los profesores de esta disciplina pueden mejorar "la competencia lectora de los alumnos haciendo hincapié en las estructuras lingüísticas específicas que son fundamentales para la comprensión de un texto de matemáticas" (Comisión Europea/EACEA/Eurydice, 2012, p. 28). Ahora bien, desarrollar actividades que promuevan aprendizajes transversales traspasando los límites de las áreas y materias tradicionales requiere una previa posición compartida sobre cuál va a ser la responsabilidad y la contribución de cada una de ellas a la adquisición de esas competencias.

Por otra parte, es sabido que las competencias suelen ser difícilmente evaluables mediante las herramientas tradicionales, pensadas para medir únicamente la adquisición de conocimientos disciplinares. La evaluación de las competencias transversales requiere nuevos instrumentos que permitan valorar los logros alcanzados por el alumnado a través de las diferentes materias. Además, teniendo en cuenta que las competencias combinan conocimientos, capacidades y actitudes, su adquisición solo puede comprobarse en el marco de un contexto determinado (Scallon, 2007). Esta circunstancia resulta especialmente relevante a propósito de las competencias sociales y cívicas, para cuya evaluación se puede proponer al alumnado varias situaciones problemáticas de la vida real con el objeto de que se manifiesten sobre cómo habrían reaccionado o cómo se habrían comportado en el caso de que se vieran implicados en ellas.

Además, en el ámbito de la educación informal (tanto en actividades escolares no curriculares como fuera de la escuela) también surgen problemas sociales relevantes susceptibles de ser aprovechados para evaluar la adquisición de competencias sociales y cívicas. Lamentablemente, a este respecto estamos muy lejos aún de lo que ocurre en muchos países de nuestro contexto:

La participación activa de los estudiantes en las actividades escolares y comunitarias es un elemento esencial de las competencias sociales y cívicas en la gran mayoría de países europeos y un tercio de ellos tienen en cuenta esta participación en las evaluaciones sumativas (...). La evaluación de la participación del alumnado se desarrolla en diferentes formatos, entre ellos el registro de perfiles personales o la validación de la contribución del alumno a la vida escolar mediante un certificado final y el reconocimiento de la participación en actividades extraescolares orientadas a la comunidad. Otros enfoques tienen en cuenta criterios relativos a la participación activa en discusiones y debates a nivel escolar, la evaluación en una materia que tenga relación con la ciudadanía o calificaciones en comportamiento. (Comisión Europea/EACEA/Eurydice, 2012, p. 34).

\section{CONCLUSIÓN}

Parece como si todos los problemas que afloran en nuestra sociedad tuviesen una fácil, rápida y mágica solución, que consiste en la creación de una nueva materia escolar, desde la educación nutricional hasta el ajedrez, pasando por la oratoria, la seguridad vial, la educación emocional, el emprendimiento, la educación financiera, el uso responsable del teléfono móvil y las redes sociales... 
Pero la realidad es que no son necesarias nuevas materias escolares; al contrario, sobran las que hay o, al menos, buena parte de ellas: todas las que no sirven para que el alumnado aprenda. Es necesario trabajar en el aula de forma más competencial, de modo que los aprendizajes sean útiles para resolver problemas y para desenvolverse en la sociedad de la que formamos parte.

La compartimentación del saber escolar en cajones herméticos y sesiones de clase cronometradas de un ajustado número de minutos dificulta la adquisición de aprendizajes relevantes y solo potencia la transmisión de contenidos académicos. Se debe priorizar la calidad de la educación en vez de la cantidad: enseñar menos y aprender más; potenciar la creatividad y el espíritu crítico, y no la superación de pruebas de evaluación.

Las nuevas pedagogías (en realidad, no tan nuevas) apuestan por la supresión total o parcial de las asignaturas, sustituidas por grandes ámbitos en los que tenga cabida el trabajo por proyectos, que persigue objetivos tanto personales como grupales y que se modula alrededor del aprendizaje cooperativo para alcanzar un determinado resultado práctico. En la misma dirección, otras estrategias que posibilitan el desarrollo de las competencias clave se centran en la utilización del portfolio, el aprendizaje basado en problemas, el aprendizaje-servicio y las comunidades de aprendizaje. Todas ellas favorecen los aprendizajes significativos, permiten la aplicación del conocimiento en situaciones prácticas, facilitan una evaluación formativa integrada en el propio proceso de enseñanza-aprendizaje y promueven las medidas inclusivas de atención a la diversidad.

Claro que este envite requiere abordar modificaciones de gran calado en la organización escolar, ya que alteran profundamente prácticas y rutinas muy arraigadas en nuestro contexto, como son la hegemonía del libro de texto, el uso rígido de tiempos y espacios, las pruebas formales de evaluación, el número de profesores presentes en el aula, la implicación (que no injerencia) de los padres en la educación de sus hijos y la propia formación del profesorado.

\section{REFERENCIAS}

Audigier, F. (1997). Histoire et géographie: un modèle disciplinaire pour penser l'identité professionnelle. Recherche et Formation, (25), pp. 9-21. DOI: https://doi.org/10.3406/refor.1997.1421

Barragán Medero, F. (1991). Educación sexual y Reforma. Cuadernos de Pedagogía, (190), pp. 52-53.

Busquets, M. D. y Leal, A. (1993). La educación para la salud. En M. D. Busquets y otros, Los temas transversales. Claves de la formación integral (pp. 45-80). Madrid: Santillana.

Cainzos, M. (1993). El consumo como tema transversal. En M. D. Busquets y otros, Los temas transversales. Claves de la formación integral. Madrid: Santillana, pp. 81-115.

Chervel, A. (1991). Historia de las disciplinas escolares. Reflexiones sobre un campo de investigación. Revista de Educación, (295), pp. 59-111.

Chervel, A. (1998). La culture scolaire. Une approche historique. París: Belin. 
Cidad Maestro, E. (1991). Perspectivas sobre educación del consumidor. Madrid: Instituto Nacional del Consumo (Ministerio de Sanidad y Consumo).

Clemente Cubillas, E. (1992). Educación Ambiental y Administraciones Públicas. Boletín de la Asociación de Geógrafos Españoles, (14), pp. 23-28.

Comisión Europea (2007). Competencias clave para el aprendizaje permanente. Un marco de referencia europeo. Luxemburgo: Oficina de Publicaciones Oficiales de las Comunidades Europeas. Disponible en: https://www.educacionyfp.gob.es/dctm/ministerio/educacion/ $\mathrm{mecu} / \mathrm{movilidad}$-europa/competenciasclave.pdf?documentId=0901e72b80685fb1

Comisión Europea/EACEA/Eurydice (2012). El desarrollo de las competencias clave en el contexto escolar en Europa: desafios y oportunidades para la politica en la materia. Informe de Eurydice. Luxemburgo: Oficina de Publicaciones de la Unión Europea. DOI: https:// doi.org/10.2797/13938

Forquin, J. C. (1988). École et culture. Le point de vue des sociologues britanniques. Bruselas: De Boeck-Wermael.

González Lucini, F. (1994). Temas transversales y áreas curriculares. Madrid: Anaya.

Lederach, J. P. (1984). Educar para la paz. Objetivo escolar. Barcelona: Fontamara.

Macía-Arce, X. C.; Rodríguez-Lestegás, F. y Armas-Quintá, F. X. (2018). El desarrollo de la competencia social y cívica en el currículo de Geografía. Revista Científica da Faculdade de Balsas, 9(1), pp. 114-122. Disponible en: http://revista.unibalsas.edu.br/index.php/ unibalsas/article/view/215/133

Moreno, A. (1986). Cómo se enseña a ser niña: el sexismo en la escuela. Barcelona: Icaria.

Moreno, M. (1993). Los temas transversales: una enseñanza mirando hacia delante. En M. D. Busquets y otros, Los temas transversales. Claves de la formación integral (pp. 9-43). Madrid: Santillana.

Muñoz Jiménez, J. (1992). Perspectiva ambiental e integración disciplinar en Geografía. Boletín de la Asociación de Geógrafos Españoles, (14), pp. 1-6.

Novo, M. (1995). La educación ambiental. Bases éticas, conceptuales y metodológicas. Madrid: Universitas.

Pujol, R. M. (1996). Educación y consumo. La formación del consumidor en la escuela. Barcelona: ICE de la Universidad de Barcelona/Horsori.

Reyzábal, M. V. y Sanz, A. I. (1995). Los ejes transversales. Aprendizajes para la vida. Madrid: Escuela Española.

Rodríguez Jares, X. (1991). Educación para la paz. Su teoría. Su práctica. Madrid: Popular.

Rodríguez Lestegás, F. (1994). A contribución das Ciencias Sociais á transversalidade no currículo. Revista Galega do Ensino, (5), pp. 103-115.

Rodríguez Lestegás, F. (1997). La Geografía en el nuevo Bachillerato: su valor formativo como disciplina y su contribución a la transversalidad. Innovación Educativa, (7), pp. 209-224. Recuperado de http://hdl.handle.net/10347/5250

Rodríguez Lestegás, F. (2000). La elaboración del conocimiento geográfico escolar: ¿de la ciencia geográfica a la geografía que se enseña o viceversa? Íber. Didáctica de las Ciencias Sociales, Geografía e Historia, (24), pp. 107-116. 
Rodríguez Lestegás, F. (2002). Concebir la geografía escolar desde una nueva perspectiva: una disciplina al servicio de la cultura escolar. Boletín de la Asociación de Geógrafos Españoles, (33), pp. 173-186.

Rodríguez Lestegás, F. (2003). Los documentos cartográficos como ejercicios-tipo para el control del conocimiento geográfico escolar. Boletín de la Asociación de Geógrafos Españoles, (35), pp. 263-271.

Sanz López, C. y Sánchez Alhama, J. (1995). Medio ambiente y sociedad. De la metáfora organicista a la preservación ecológica. Granada: Comares.

Scallon, G. (2007). L'évaluation des apprentissages dans une approche par compétences. Bruselas: Be Boeck. 